\title{
Additive Utility with Intransitive Indifference and without Independence: A Homogeneous Case
}

\author{
Marc Le Menestrel $^{*}$ and Bertrand Lemaire ${ }^{\dagger}$
}

June 2002

\begin{abstract}
In the homogeneous case of one type of goods or objects, we prove the existence of an additive utility function without assuming transitivity of indifference and independence. The representation reveals a positive factor $\alpha \leq 1$ that influences rational choice beyond the utility function and explains departures from these standard axioms of utility theory $(\alpha=1)$.
\end{abstract}

Keywords: Rationality, Utility, Maximization.

JEL: A0, B4, C0, D0.

*Universitat Pompeu Fabra, Departament d'Economia i Empresa, Barcelona, España.

†CNRS, Université Paris-Sud, Département de Mathématiques, Orsay, France. 


\section{Introduction}

Standard theories of utility can be formulated as a collection of axioms about a nonempty ordering $\succ$ on a set $A$ and a binary (commutative, associative) operation $\circ$ on $A$ that permit the construction of a real-valued function $u$ on $A$ verifying

$$
\begin{aligned}
& x \succ y \Longleftrightarrow u(x)>u(y), \\
& u(x \circ y)=u(x)+u(y) .
\end{aligned}
$$

Two groups of axioms are crucial to these theories. Firstly, the ordering is assumed to be asymmetric: $x \succ y \Rightarrow y \nsucc x$, and negatively transitive: $(x \nsucc y$ and $y \nsucc z) \Rightarrow x \nsucc z$. Note that these two properties imply that the ordering is also transitive: $(x \succ y$ and $y \succ z) \Rightarrow x \succ z$. Secondly, the combination of the ordering and the operation is assumed to verify a form of independence or cancellation law, also called monotonicity: $x \succ y \Leftrightarrow(x \circ z \succ$ $y \circ z$ for all $z \in A$ ). Note that this property of independence, joint to the asymmetry of the ordering, imply that the operation is $\succ$-regular: $(x \succ y$ or $y \succ x) \Rightarrow(x \circ z \neq y \circ z$ for all $z \in A)$. If there exists a real-valued function $u$ on $A$ verifying $(i)$ and $(i i)$, then all these axioms necessarily hold (because they hold for the triple $\langle\mathbb{R},>,+\rangle$ ). In this sense, if a theory replaces negative transitivity with the weaker axiom of transitivity, allowing intransitive indifference, then $(i)$ must be modified in

$$
x \succ y \Longrightarrow u(x)>u(y) .
$$

On the other hand, if a theory relaxes independence maintaining a twoway representation like $(i)$, then $(i i)$ cannot be satisfied. Those theories lose the additivity of the utility function. In both examples, the theory is significantly weakened. ${ }^{1}$

\footnotetext{
${ }^{1}$ For a presentation of the standard theory, see e.g. Fishburn (1970a); Krantz \& Al. (1971); Barbera \& Al. (1998). On the independence condition in preference theory, see Fishburn \& Wakker (1995). A seminal reference on intransitive indifference is Luce (1956). For a review of intransitive indifference in preference theory: Fishburn (1970b) and also Krantz \& Al. (1971). For the treatment of discrimination through interval orders, see e.g. Fishburn (1985). About additivity, see for instance Wakker (1988a); Luce \& Al. (1990, Chap. 19). About empirical deviations from standard utility theory, see for instance Hogarth \& Reder (1987); Kahneman \& Tversky (2000).
} 
Assuming transitivity (i.e. without assuming negative transitivity) and replacing independence by a weaker property (replicated independence, see Definition 1), we would show there exists a utility function $u$ that verifies (ii) and a two-way representation $\left(i^{\prime \prime}\right)$ more general than $(i)$. More precisely, we expect there exists a function $\alpha: A \times A \rightarrow \mathbb{R}_{>0}$ (satisfying certain technical conditions ensuring the uniqueness of the pair $(u, \alpha)$ up to scalar) such that

$$
x \succ y \Longleftrightarrow \alpha(x, y) u(x)>u(y) .
$$

In a discrete and homogeneous case (see Definition 1, section 2), we prove here that $\alpha$ is a constant $\leq 1$ (in this case, no "technical condition" is needed). Further, we slightly generalize this result to a continuous setting (section 3).

With this model, we can, for instance, reflect a rational individual being indifferent between $€ 100$ and $€ 101$, and between $€ 101$ and $€ 102$, while strictly preferring $€ 102$ to $€ 100$. Moreover, an individual who is indifferent between $€ 101$ and $€ 102$ may not be indifferent between $€ 1$ and $€ 2$. Therefore, such a model allows one to reflect a lack of discrimination (intransitive indifference) and a diminishing marginal utility (violation of independence). For the factor $\alpha$, we have had in mind a model of rational behavior that combines processes and consequences. In this interpretation, $\alpha$ would reflect intrinsic procedural concerns outside the utility function. Without doubt, other interpretations are possible. ${ }^{2}$

\section{$2 \quad$ Utility Representation (Discrete Setting)}

We start with three primitives: a nonempty set $A$, a nonempty binary relation $\succ$ on $A$, and a closed binary relation $\circ$ on $A$. We write $x \sim y$ if and only if $(x \nsucc y$ and $y \nsucc x)$, and $x \succsim y$ if and only if $(x \succ y$ or $x \sim y)$. We note $\mathbb{N}_{>0}$ the set of positive integers, $\mathbb{Q}_{>0}$ the set of positive rational numbers and $\mathbb{R}_{>0}$ the set of positive real numbers.

Definition 1 Let $A$ be a nonempty set, $\succ$ a nonempty binary relation on $A$, and $\circ$ a closed binary operation on $A$. The triple $\langle A, \succ, \circ\rangle$ is a partially ordered positive structure if and only if the following five axioms are satisfied for all $x, y, z \in A$ :

\footnotetext{
${ }^{2}$ For the relevance of a procedural dimension in rationality, see e.g. Simon (1978) and Sen (1997). A model of rational behavior combining processes and consequences is tentatively explored in Le Menestrel (1999, 2001a, 2001b). See also Le Menestrel \& Van Wassenhove (2001). For a resembling (proportional) lack of discrimination in psychology (see e.g. Suppes \& Al. 1989).
} 
1. Strict Partial Order: $x \succ y \Rightarrow y \nsucc x ;(x \succ y$ and $y \succ z) \Rightarrow x \succ z$.

2. Commutativity; Associativity: $x \circ y=y \circ x ;(x \circ y) \circ z=x \circ(y \circ z)$.

3. Positivity: $x \succ y \Longrightarrow x \circ z \succ y$.

4. Replicated Independence: $x \succ y \Leftrightarrow\left(n x \succ n y\right.$ for all $\left.n \in \mathbb{N}_{>0}\right)$, where $n x$ is defined inductively by $1 x=x$ and $(n+1) x=n x \circ x$.

5. Archimedean: If $x \succ y$, then there exists $n \in \mathbb{N}_{>0}$ such that $n x \succ$ $(n+1) y$.

A partially ordered positive structure $\langle A, \succ, \circ\rangle$ is said to be homogeneous if it satisfies the following condition, for all $x, y \in A$ :

6. Homogeneity: $m x=n y$ for some $(m, n) \in \mathbb{N}_{>0} \times \mathbb{N}_{>0}$.

A nonempty set $A$ endowed with a closed associative and commutative binary operation $\circ$, is called a commutative semigroup. ${ }^{3}$ A commutative semigroup $A$ is said to be regular (respectively replicated-regular) if for all $x \in A$, the map $A \rightarrow A, y \longmapsto x \circ y$ (respectively the map $\mathbb{N}_{>0} \rightarrow A, n \longmapsto n x$ ) is injective. Let $\langle A, \succ, \circ\rangle$ be a partially ordered positive structure. Then (by replicated independence and asymmetry) the commutative semigroup $A$ is replicated- $\succ$-regular: $(x \succ y$ or $y \succ x) \Rightarrow(n x \neq n y$ for all $n \in \mathbb{N})$. Clearly, the four notions of regularity we have introduced in this paper satisfy the following implications:

$$
\text { regularity } \Rightarrow \succ \text {-regularity } \Rightarrow \text { replicated- } \succ \text {-regularity, }
$$

and

$$
\text { regularity } \Rightarrow \text { replicated-regularity } \Rightarrow \text { replicated- } \succ \text {-regularity. }
$$

It is not difficult to verify (see the proof of Theorem 1 below) that if $A$ is homogeneous, then it is also replicated-regular. In particular (always assuming $A$ is homogeneous), this implies that for all $x, y \in A$, the set $\left\{\frac{m}{n}: m, n \in \mathbb{N}_{>0}, m x=n y\right\}$ is reduced to one element.

\footnotetext{
${ }^{3}$ See Fuchs (1963) for a seminal algrebraic treatment. There, axiom 5 is said to exclude "anomalous" pairs. It has been introduced by Alimov in 1950, see reference above (p. 162s) and also footnote 4 below. The name for axiom 4 has been suggested to us by Peter Fishburn.
} 
Theorem 1 Let $\langle A, \succ, \circ\rangle$ be a partially ordered positive homogeneous structure. Then there exist a function $u: A \rightarrow \mathbb{R}_{>0}$ and a real number $0<\alpha \leqslant 1$ such that for all $x, y \in A$

$$
\begin{gathered}
x \succ y \Longleftrightarrow \alpha u(x)>u(y), \\
u(x \circ y)=u(x)+u(y) .
\end{gathered}
$$

If $(v, \beta)$ is another pair satisfying $\left(i^{\prime \prime}\right)$ and $(i i)$, then $\beta=\alpha$ and there exists a real number $\lambda>0$ such that $v=\lambda u$. Moreover, $u$ is injective if and only if $A$ is regular, $u$ can be chosen with values in $\mathbb{Q}_{>0}$, and $\alpha \in \mathbb{Q}$ if and only if there exist $x, y \in A$ such that $\alpha u(x)=u(y)$.

Proof. Since $\succ$ is not empty, there exist $x, y \in A$ such that $x \succ y$. Let $z, z^{\prime} \in A$, and choose $(m, n),\left(m^{\prime}, n^{\prime}\right) \in \mathbb{N}_{>0} \times \mathbb{N}_{>0}$ such that $m x=n z$ and $m^{\prime} y=n^{\prime} z^{\prime}$ (homogeneity). By replicated independence, we have $m^{\prime} m x \succ$ $m m^{\prime} y$, i.e. $p z \succ q z^{\prime}$ with $p=m^{\prime} n$ and $q=m n^{\prime}$. Take $z=z^{\prime}$, and suppose there exists $(a, b) \in \mathbb{N}_{>0} \times \mathbb{N}_{>0}$ such that $a>b$ and $a z=b z$. Then we have $(b+k(a-b)) z=b z$ for all $k \in \mathbb{N}_{>0}$, hence $m^{\prime \prime}(b+k(a-b)) z=m^{\prime \prime} b z$ for all $\left(m^{\prime \prime}, k\right) \in \mathbb{N}_{>0} \times \mathbb{N}_{>0}$. Taking $m^{\prime \prime}=q$, we can choose $k$ big enough so that $q(b+k(a-b))>p b$. Since $p b z \succ q b z$ (replicated independence), by positivity we obtain $q(b+k(a-b)) z \succ q b z$, which is impossible. This implies the replicated-regularity of $A$.

For $x \in A$, we define the subsets of $\mathbb{Q}_{>0}$

$$
\begin{aligned}
& \mathcal{Q}_{x}=\left\{\frac{m}{n}: m x \succsim n x, \exists(m, n) \in \mathbb{N}_{>0} \times \mathbb{N}_{>0}\right\}, \\
& \mathcal{P}_{x}=\left\{\frac{m}{n}: m x \succ n x, \exists(m, n) \in \mathbb{N}_{>0} \times \mathbb{N}_{>0}\right\} .
\end{aligned}
$$

By homogeneity and replicated independence, for all $x, y \in A$, we have $\mathcal{Q}_{x}=\mathcal{Q}_{y}$ and $\mathcal{P}_{x}=\mathcal{P}_{y}$. So we can drop the index $x$ in the notation $\mathcal{Q}_{x}$ and $\mathcal{P}_{x}$. From the previous paragraph, $\mathcal{P}$ is not empty, and $1 \in \mathcal{Q}$. We also have $\mathbb{Q}_{>0}=\mathcal{Q} \cup \mathcal{P}^{-1}=\mathcal{Q}^{-1} \cup \mathcal{P}$ and $\mathcal{Q} \cap \mathcal{P}^{-1}=\mathcal{Q}^{-1} \cap \mathcal{P}=\varnothing$.

By positivity and replicated independence, we have $q \in \mathcal{Q} \Rightarrow \mathbb{Q}_{\geq q} \subset \mathcal{Q}$ and $q \in \mathcal{P} \Rightarrow \mathbb{Q}_{\geq q} \subset \mathcal{P}$.

We define $r=\inf _{\mathbb{R}} \mathcal{Q}$ and $s=\inf _{\mathbb{R}} \mathcal{P}$.

Because $1 \in \mathcal{Q}$, we have $0 \leq s \leq 1$. Because of positivity, we have $r \geq 1$.

If $s=0$, then for all $(m, n) \in \mathbb{N}_{>0} \times \mathbb{N}_{>0}$, there exists $\left(m^{\prime}, n^{\prime}\right) \in \mathbb{N}_{>0} \times \mathbb{N}_{>0}$ such that $\left(m^{\prime}, n^{\prime}\right) \in \mathcal{Q}$ and $\frac{m^{\prime}}{n^{\prime}}<\frac{m}{n}$. Hence $\frac{m}{n} \in \mathcal{Q}$. Therefore $\mathcal{P}=\varnothing$, contradiction. Hence $0<s \leq 1$. The same argument implies that $\mathbb{Q}_{>s} \subset \mathcal{Q}$. 
Suppose $s \in \mathbb{Q} \backslash \mathcal{Q}$. Take $(m, n) \in \mathbb{N}_{>0} \times \mathbb{N}_{>0}$ such that $s=\frac{m}{n}$. Since $s \notin \mathcal{Q}$, we have $n x \succ m x$ and thus $p n x \succ(p+1) m x$ for some $p \in \mathbb{N}_{>0}$ (Archimedean). Therefore $\frac{(p+1)}{p} s \notin \mathcal{Q}$ which contradicts $\mathbb{Q}_{>s} \subset \mathcal{Q}$. Therefore, $s \in \mathbb{Q}$ implies $s \in \mathcal{Q}$.

Finally, we have $\mathcal{Q}=\mathbb{Q}_{\geq s}$, and also $\mathcal{P}=\mathbb{Q}_{>\frac{1}{s}}$. Hence, $r=\frac{1}{s}$.

By replicated-regularity, for all $x, y \in A$, there exists a unique $q_{x, y} \in \mathbb{Q}_{>0}$ such that $\left\{\frac{m}{n}: m, n \in \mathbb{N}_{>0}, m x=n y\right\}=\left\{q_{x, y}\right\}$. Let $x \in A$. We define a function $f_{x}: A \longrightarrow \mathbb{Q}_{>0}$ by $f_{x}=q_{x, y}$. Let $y, y^{\prime} \in A$. We write $m x=n y$ and $m^{\prime} x=n^{\prime} y^{\prime}$ for some $(m, n),\left(m^{\prime}, n^{\prime}\right) \in \mathbb{N}_{>0} \times \mathbb{N}_{>0}$. Since $\left(n^{\prime} m+n m^{\prime}\right) x=$ $n n^{\prime}\left(y \circ y^{\prime}\right)$, we have $q_{x, y \circ y^{\prime}}=\frac{n^{\prime} m+n m^{\prime}}{n n^{\prime}}=\frac{m}{n}+\frac{m^{\prime}}{n^{\prime}}$, i.e. $f_{x}\left(y \circ y^{\prime}\right)=f_{x}(y)+f_{x}\left(y^{\prime}\right)$. Moreover,

$$
y \succ y^{\prime} \Leftrightarrow n^{\prime} n y \succ n n^{\prime} y \Leftrightarrow n^{\prime} m x \succ n m^{\prime} x \Leftrightarrow \frac{n^{\prime} m}{n m^{\prime}} \in \mathcal{P} \Leftrightarrow \frac{n^{\prime} m}{n m^{\prime}}>r
$$

and

$$
\frac{n^{\prime} m}{n m^{\prime}}>r \Leftrightarrow \frac{m}{n}>r \frac{m^{\prime}}{n^{\prime}} \Leftrightarrow s f_{x}(y)>f_{x}\left(y^{\prime}\right) .
$$

So we have proved that the pair $(u, \alpha)=\left(f_{x}, s\right)$ verifies the conditions $\left(i^{\prime \prime}\right)$ and $(i i)$ of Theorem 1 . By construction $u$ is $\mathbb{Q}_{>0}$-valued.

Let $f^{\prime}: A \rightarrow \mathbb{R}_{>0}$ be a function such that $f^{\prime}(y \circ z)=f^{\prime}(y)+f^{\prime}(z)$ for all $y, z \in A$. Let $y \in A$, and write $m x=n y$ for some $(m, n) \in \mathbb{N}_{>0} \times \mathbb{N}_{>0}$. Then we have $m f^{\prime}(x)=f^{\prime}(m x)=f^{\prime}(n y)=n f^{\prime}(y)$, i.e. $f^{\prime}(y)=\lambda f_{x}(y)$ with $\lambda=f^{\prime}(x)$. Then $u$ is unique up to scaling transformation, which implies the uniqueness of $\alpha$. Condition $(i i)$ of the Theorem implies that $u$ is injective if and only if $A$ is regular. The last assertion of the Theorem is clear.

Reciprocally, if $(A, \circ)$ is a commutative semigroup (not necessarily homogeneous) endowed with a nonempty binary relation $\succ$ such that there exist a function $u: A \rightarrow \mathbb{R}$ and a real number $0<\alpha \leq 1$ satisfying the conditions $\left(i^{\prime \prime}\right)$ and $(i i)$ of Theorem 1 , then the triple $\langle A, \succ, o\rangle$ is a partially ordered positive structure. The verification of this assertion is easy and left to the reader.

Theorem 1 implies that $\alpha=1$ if and only if negative transitivity and independence hold. We recover the standard theory where $(i)$ and $(i i)$ are satisfied. 4 In general, the factor $\alpha$ may not equal to one, "twisting" the representation and preventing the interpretation that a rational individual acts as if he maximizes the utility function $u$.

\footnotetext{
${ }^{4}$ When $\alpha=1$, the triple $\langle A, \succ,+\rangle$ is a closed positive extensive structure as defined by Krantz and Al. (1970, p. 73). With respect to the Archimedean axiom used there and axiom 5 here, see the discussion referred to in footnote 3 .
} 


\section{A Continuous Setting Generalization}

Formulated using a discrete algebraic approach, Theorem 1 can be generalized to a continuous set of goods or objects. Retaining the algebraic approach, we now introduce such a generalization. ${ }^{5}$

Let $R \subset \mathbb{R}_{>0}$ be a subset containing 1 such that for all $\lambda, \mu \in R$, we have $\lambda+\mu \in R, \lambda \mu \in R$, and $\lambda>\mu \Rightarrow \lambda-\mu \in R$. Since $1 \in R$, we have $\mathbb{N}_{>0} \subset R$. We call $R$ - semimodule a commutative semigroup $(A, \circ)$ endowed with a closed operation $R \times A \rightarrow A,(\lambda, \mu) \mapsto \lambda \cdot \mu$ such that for all $x, y \in A$ and $\lambda, \mu \in R$, we have:

$$
\begin{aligned}
& \lambda \cdot(x \circ y)=(\lambda \cdot x) \circ(\lambda \cdot y), \\
& (\lambda+\mu) \cdot x=(\lambda \cdot x) \circ(\mu \cdot x), \\
& \lambda \cdot(\mu \cdot x)=(\lambda \mu) \cdot x, \\
& 1 \cdot x=x .
\end{aligned}
$$

Because of the last condition, for $n \in \mathbb{N}_{>0}$, we have $n \cdot x=n x$. Therefore, the notions of commutative semigroup and $\mathbb{N}_{>0}$-semimodule coincide. An $R$-semimodule $(A, \circ, \cdot)$ is said to be $R$-regular if for all $x \in A$ the map $R \rightarrow A, \lambda \mapsto \lambda \cdot x$ is injective.

Definition 2 Let $A$ be a nonempty set, $\succ$ a nonempty binary relation on $A$, ○ a closed binary operation on $A$, and - a closed operation of $R$ on $A$. The quadruple $\langle A, \succ, \circ, \cdot\rangle$ is a partially ordered positive $R$-structure if and only if the following five axioms are satisfied for all $x, y \in A$ :

1. Strict Partial Order (Definition 1, axiom 1).

2. $(A, \circ, \cdot)$ is a $R$-semimodule.

3. Positivity (Definition 1, axiom 3).

4. $R$-independence: $x \succ y \Leftrightarrow(\lambda x \succ \lambda y$ for all $\lambda \in R)$.

5. $R$-archimedean: If $x \succ y$, then there exist $\lambda, \mu \in R$ with $\lambda<\mu \in R$, such that $\lambda \cdot x \succ \mu \cdot y$.

A partially ordered positive $R$-structure $\langle A, \succ, \circ, \cdot\rangle$ is said to be homogeneous if it satisfies the following condition, for all $x, y \in A$ :

6. $R$-homogeneity: $\lambda \cdot x=\mu \cdot y$ for some $(\lambda, \mu) \in R \times R$.

\footnotetext{
${ }^{5}$ The algebraic approach is slightly more general than the topological one (see Wakker 1988b).
} 
Let $F(R) \subset \mathbb{R}_{>0}$ be the subset defined by $F(R)=\left\{\frac{\lambda}{\mu}: \lambda, \mu \in R\right\}$. Since $1 \in R$, we have the inclusions $\mathbb{N}_{>0} \subset R \subset F(R)$. And for all $\lambda, \mu \in F(R)$, we have $\lambda+\mu \in F(R), \lambda \mu \in F(R)$, and $\lambda>\mu \Rightarrow \lambda-\mu \in F(R)$. In particular, we have:

- if $R \subset \mathbb{Q}$, then $F(R)=\mathbb{Q}_{>0}$,

- if $R=\mathbb{R}_{>0}$, then $F(R)=\mathbb{R}_{>0}$.

Theorem 2 Let $\langle A, \succ, \circ, \cdot\rangle$ be a partially ordered positive homogeneous $R$-structure. Then there exist a function $u: A \rightarrow \mathbb{R}_{>0}$ and a real number $0<\alpha \leqslant 1$ such that for all $x, y \in A$ and $\lambda \in R$, we have

$$
\begin{gathered}
x \succ y \Longleftrightarrow \alpha u(x)>u(y), \\
u(x \circ y)=u(x)+u(y), \\
u(\lambda \cdot x)=\lambda u(x) .
\end{gathered}
$$

If $(v, \beta)$ is another pair satisfying $\left(i^{\prime \prime}\right),($ ii $)$ and $(i i i)$, then $\beta=\alpha$ and there exists a real number $\gamma>0$ such that $v=\gamma u$. Moreover, $u$ is injective if and only if the semigroup $(A, \circ)$ is regular, $u$ can be chosen with values in $F(R)$, and $\alpha \in F(R)$ if and only if there exist $x, y \in A$ such that $\alpha u(x)=$ $u(y)$.

Proof. Roughly speaking, it suffices to replace $\mathbb{N}_{>0}$ by $R$ and $\mathbb{Q}_{>0}$ by $F(R)$ in the proof of Theorem 1 . We sketch this briefly. Let $z, z^{\prime} \in A$. Since $\succ$ is nonempty, by $R$-homogeneity and $R$-independence, there exist $\lambda, \mu \in R$ such that $\lambda \cdot z \succ \lambda \cdot z^{\prime}$. Take $z=z^{\prime}$, and suppose there exists $(a, b) \in R \times R$ such that $a>b$ and $a \cdot z=b \cdot z$. Since $a-b \in R$, for all $k \in \mathbb{N}_{>0}$, we have $(b+k(a-b)) \cdot z=b \cdot z$. Choosing $k$ big enough so that $\mu(b+k(a-b))>\lambda b$, by $R$-independence and positivity, we obtain $\mu(b+k(a-b)) \cdot z \succ \mu b \cdot z$, which is impossible. This implies the $R$-regularity of the $R$-semimodule $(A, \circ, \cdot)$.

For $x \in A$, we define the (nonempty) subsets of $F(R)$

$$
\begin{aligned}
& \mathcal{Q}_{x}=\left\{\frac{\lambda}{\mu}: \lambda \cdot x \succsim \mu \cdot x, \exists(\lambda, \mu) \in R \times R\right\}, \\
& \mathcal{P}_{x}=\left\{\frac{\lambda}{\mu}: \lambda \cdot x \succ \mu \cdot x, \exists(\lambda, \mu) \in R \times R\right\} .
\end{aligned}
$$


By $R$-homogeneity and $R$-independence, we can drop the index $x$ in the notation $\mathcal{Q}_{x}$ and $\mathcal{P}_{x}$. We have $F(R)=\mathcal{Q} \cup \mathcal{P}^{-1}=\mathcal{Q}^{-1} \cup \mathcal{P}$ and $\mathcal{Q} \cap \mathcal{P}^{-1}=$ $\mathcal{Q}^{-1} \cap \mathcal{P}=\varnothing$. By positivity and $R$-independence, we have $q \in \mathcal{Q} \Rightarrow F(R)_{\geq q} \subset$ $\mathcal{Q}$ and $q \in \mathcal{P} \Rightarrow F(R)_{\geq q} \subset \mathcal{P}$. We define $r=\inf _{\mathbb{R}} \mathcal{Q}$ and $s=\inf _{\mathbb{R}} \mathcal{P}$. Because $1 \in \mathcal{Q}$, we have $0 \leq s \leq 1$, and because $\succ$ is nonempty, we have $s>0$ and $F(R)_{>s} \subset \mathcal{Q}$. This last inclusion, joint to the $R$-archimedean axiom, implies that if $s \in F(R)$, then $s \in \mathcal{Q}$. So we have $\mathcal{Q}=F(R)_{\geq s}, \mathcal{P}=F(R)_{\geq s^{-1}}$ and $r=s^{-1}$.

By $R$-regularity, for all $x, y \in A$, there exists a unique $q_{x, y} \in F(R)$ such that $\left\{\frac{\lambda}{\mu}: \lambda, \mu \in R, \lambda \cdot x=\mu \cdot y\right\}=\left\{q_{x, y}\right\}$. Let $x \in A$. We define a function $f_{x}: A \longrightarrow F(R)$ by $f_{x}(y)=q_{x, y}$. As in the proof of Theorem 1, we verify that the pair $(u, \alpha)=\left(f_{x}, s\right)$ verifies the conditions $\left(i^{\prime \prime}\right)$ and $(i i)$. By construction $u$ is $F(R)$-valued and $u(\lambda \cdot x)=\lambda f_{x}(y)(\lambda \in R, y \in A)$. The uniqueness of $u$ up to scaling transformation is obtained as in the proof of Theorem 1, using $R$-homogeneity and condition ( $i i i)$. All the remaining assertions of Theorem 2 are clear.

Finally, if $(A, \circ, \cdot)$ is a $R$-semimodule (not necessarily $R$-homogeneous) endowed with a nonempty binary relation $\succ$ such that there exist a function $u: A \rightarrow \mathbb{R}$ and a real number $0<\alpha \leq 1$ satisfying the conditions $\left(i^{\prime \prime}\right),(i i)$, and (iii) of Theorem 2 , then the quadruple $\langle A, \succ, \circ, \cdot\rangle$ is a partially ordered positive $R$-structure.

\section{References}

Barbera, S., Hammond, P. \& C. Seidl: 1998, Handbook of Utility Theory: Volume 1 Theory and Principles, Kluwer Academic Press.

Fishburn, P. C.: 1970a, Utility Theory for Decision-Making, John Wiley and Sons.

Fishburn, P. C.: 1970b, "Intransitive Indifference in Preference Theory: A Survey," Operations Research 18: 207-228.

Fishburn, P. C.: 1985, Interval Orders and Interval Graphs, Wiley.

Fishburn, P. C. \& P. Wakker: 1995, "The Invention of the Independence Condition for Preferences," Management Science 41, 1130-1144.

Fuchs, P.: 1963, Partially Ordered Algebraic Systems, Pergamon Press.

Hogarth, R. \& M. Reder: 1987, Rational Choice: The Contrast between Economics and Psychology, The University of Chicago Press.

Kahneman, D. \& A. Tversky: 2000, Choices, Values, and Frames, Cambridge University Press.

Krantz, D., R. D. Luce, P. Suppes \& A. Tversky: 1971, Foundations of Measurement, Volume 1: Additive and Polynomial Representations, Academic 
Press.

Le Menestrel, M.: 1999, "A Model of Rational Behavior Combining Processes and Consequences," Unpublished Ph.D. Dissertation, INSEAD, France.

Le Menestrel, M.: 2001a, "A Process Approach to the Utility for Gambling," Theory and Decision 50: 249-262.

Le Menestrel, M.: 2001b, "A One-Shot Prisoners' Dilemma with Process Utility," Miméo.

Le Menestrel, M. \& L. Van Wassenhove: 2001, "The Domain and Interpretation of Utility Function: An Exploration," Theory and Decision 51: 329-349. Luce, R.D., D.H. Krantz, P. Suppes, \& A. Tversky: 1990, Foundations of Measurement, Volume 3: Representation, Axiomatization, and Invariance, Academic Press.

Luce, R.D.: 1956, "Semiorders and a Theory of Utility Discrimination," Econometrica 24: 178-191.

Sen, A.: 1997, "Maximization and the Act of Choice," Econometrica 65: 745-779.

Simon, H.A.: 1978, "Rationality as Process and as Product of Thought," The American Economic Review 68: 1-16.

Suppes, P. , D. H. Krantz, R. D. Luce and A. Tversky: 1989, Foundations of measurement, Volume 2: Geometrical, Threshold, and Probabilistic Representations, Academic Press.

Wakker, P.: 1988a, Additive Representations of Preferences, Kluwer Academic Publishers.

Wakker, P.: 1988b, "The Algebraic versus the Topological Approach to Additive Representations," Journal of Mathematical Psychology 32, 421-435. 